\title{
COVID-19 Pandemic in India: What Lies Ahead-Letter to the Editor
}

\author{
Mitra P, Misra S, Sharma P. COVID-19 Pandemic in India: What Lies Ahead [pub- \\ lished online ahead of print, 2020 Apr 20]. Indian J Clin Biochem. 2020;1-3. doi: \\ https://doi.org/10.1007/s12291-020-00886-6
}

\author{
Ashlesha Kaushik ${ }^{1} \cdot$ Sandeep Gupta $^{2} \cdot$ Mangla Sood $^{3}$
}

Received: 1 June 2020/Accepted: 3 June 2020/Published online: 10 June 2020

(C) Association of Clinical Biochemists of India 2020

To the Editor: We were pleased to read the very timely editorial in this journal entitled COVID-19 Pandemic in India: What Lies Ahead by the Mitra P et al. [1]. We would like to commend the authors for providing valuable insights into clinical epidemiology of COVID-19 and comment on the evolving situation with recent evidence, to complement the issues raised by the thought-provoking editorial.

We are witnessing a mammoth global pandemic of COVID-19, affecting 215 countries with 5,968,693 cases and 365,796 deaths as of May 30, 2020 [2]. The present epicenter has shifted to the United States with $1,749,846$ cases and 102,900 deaths. SARS-CoV-2 cases in India are also increasing with $1,75,957$ cases and 4996 deaths being reported as of May 30, 2020, with Maharashtra, Tamil Nadu, Gujarat, Delhi, Rajasthan and Madhya Pradesh

Ashlesha Kaushik

ashleshakaushik@gmail.com

Sandeep Gupta

sandeep.gupta@unitypoint.org

Mangla Sood

drmanglasood@gmail.com

1 Pediatric Infectious Diseases and COVID-19 Incident Command, Unity Point Health at St. Luke's Regional Medical Center, Department of Pediatrics, University of Iowa Carver College of Medicine, 2720 Stone Park Blvd, Sioux City, IA 51104, USA

2 Pulmonary Rehabiliation, COVID-19 Critical Care Incident Command, Division of Pulmonary and Critical Care, Unity Point Health at St. Luke's Regional Medical Center, 2720 Stone Park Blvd, Sioux City, IA 51104, USA

3 Department of Pediatrics, Former State Child Health Lead, Indira Gandhi Medical College, Shimla, Himachal Pradesh 171001, India being the worst affected states [3]. Older adults are most severely affected as noted by the authors; however, novel pediatric manifestations resembling Kawasaki disease have been recently recognized, marked by elevated biochemical markers of inflammation including ferritin and D-dimers [4], labeled recently as Multisystem Inflammatory Syndrome by the World Health Organization.

While several studies are underway for vaccines and therapies for COVID-19 worldwide, a recent report showed remdesivir, a nucleoside analog that causes premature viral RNA (ribonucleic acid) chain termination thereby inhibiting viral replication, to be effective in adult patients [5]. Interleukin- 6 inhibitors like tocilizumab are also being used as therapeutic agents, given the associated cytokine storm with COVID-19 [6]. Therapy with convalescent plasma has shown promise and a Phase II, Open Label, Randomized Controlled Trial to Assess the Safety and Efficacy of Convalescent Plasma to Limit COVID-19 associated Complications in Moderate Disease (PLACID Trial) has been initiated by Indian Council of Medical Research (ICMR) in 46 hospitals within India [7].

The authors have raised several important issues for the pandemic in India. With the largest national lockdown to contain the spread of virus in the country with a population of 1.38 billion (imposed on March 25th, and later extended in four stages till May 31st 2020), we could say that from theoretically being at the highest risk of becoming the epicentre for COVID-19, India has thus far been saved from becoming the global hotspot. Until now, the case rate in India is relatively low at 2 per million, fatality rate is 3.15 per number of cases, and recovery rate is $48 \%$ [3]. The national strategy to contain the virus includes dividing state districts into three zones based on number of coronavirus cases, including Red/ Orange zones (where new cases are emerging) and Green zones (those not reporting fresh cases 
for some time) [3]; and segregating healthcare facilities across the country as Non-COVID and COVID facilities. Three types of dedicated COVID facilities include COVID care centers (for mild or suspected cases), COVID Health centers (for moderate cases with basic life support facilities) and COVID Hospitals (with fully equipped Intensive care units with ventilators and oxygen support for sick patients). Thus far, a total of 36, 11,599 patient-samples have been tested for COVID-19 in India [3]. ICMR testing protocol recommends reverse transcription polymerase chain reaction as the confirmatory diagnostic test, however, we agree with the authors that antibody testing would be valuable for population-wide use for delineating people at risk for disease transmission as well as acquisition. Presently, however, antibody testing is neither widespread nor utilized except for pooled sampling.

Till now, COVID-19 in India has been addressed predominantly by containment measures (quarantine, isolation of infected individuals, contact- tracing and reducing movement of people in areas with high case-loads). However, with the recent rise in number of infected cases, it is now time to shift focus from containment to community mitigation, also known as 'flattening the curve' in epidemiological terms. Potential interventions include social distancing, minimizing unnecessary exposure of vulnerable population (individuals aged over 60 years, those with cardiovascular disease, diabetes, chronic respiratory disease and cancer) by helping them stay at home, and education of the masses. With planned relaxation of lockdown in the coming days, implementing guidelines to prevent COVID-19 infection at workplace and containment zones would be paramount, including travel advisories, increased use of tools like Arogya Setu app for contact tracing and encouraging tele-consultations [3]. We concur with the authors that an increase in the capacity for testing and treatment, isolating confirmed cases, and tracing new cases is needed to disrupt the transmission chain, and seems to be the way forward in tackling this infection, till an effective vaccine or cure becomes available.

\section{Acknowledgements None}

Author contributions All authors contributed to the study conception and design. Material preparation, data collection and analysis were performed by Ashlesha Kaushik, Sandeep Gupta, Mangla Sood. The first draft of the manuscript was written by Ashlesha Kaushik and all authors commented on previous versions of the manuscript. All authors have read and approved the final manuscript. AK: contributed to the concept, design, definition of intellectual content, literature search, manuscript preparation, manuscript editing and manuscript review. SG: contributed to the definition of intellectual content, literature search, manuscript preparation, manuscript editing and manuscript review. MS: contributed to the definition of intellectual content, literature search, manuscript preparation, manuscript editing and manuscript review.

Funding None.

\section{Compliance with ethical standards}

Conflict of interest The authors declare that there is no conflict of interests/competing interests.

\section{References}

1. Mitra P, Misra S, Sharma P. COVID-19 Pandemic in India: What lies ahead [published online ahead of print, 2020 Apr 20]. Indian J Clin Biochem. 2020. https://doi.org/10.1007/s12291-020-00886-6.

2. World Health Organization novel Coronavirus 2019. https://www. who.int/emergencies/diseases/novel-coronavirus-2019. Last Accessed 30 May 2020

3. Ministry of Health and Family Welfare, Government of India. COVID-19 cases in India. https://www.mohfw.gov.in. Last Accessed 30 May 2020

4. Verdoni L, Mazza A, Gervasoni A, Martelli L, Ruggeri M, Ciuffreda M, Bonanomi E, D'Anitga L. An outbreak of severe Kawasaki-like disease at the Italian epicentre of the SARS-CoV-2 epidemic: an observational cohort study. Lancet. 2020. https://doi. org/10.1016/S0140-6736(20)31129-6.

5. Grein J, Ohmagari N, Shin D, Diaz G, Asperges E, Castagna A, et al. Compassionate Use of Remdesivir for Patients with Severe Covid-19. N Engl J Med. 2020. https://doi.org/10.1056/ NEJMoa2007016.

6. Xu X, Han M, Li T, et al. Effective treatment of severe COVID-19 patients with tocilizumab. Proc Natl Acad Sci USA. 2020;117(20): 10970-5. https://doi.org/10.1073/pnas.2005615117

7. Indian Council of Medical Research. COVID-19 disease updates. Available from: https://main.icmr.nic.in/content/covid-19. Last Accessed 30 May 2020

Publisher's Note Springer Nature remains neutral with regard to jurisdictional claims in published maps and institutional affiliations. 